\title{
Acción de los antiagregantes plaquetarios sobre el recuento de plaquetas en el plasma rico en plaquetas
}

\author{
Antiplatelet action over the platelet count in platelet-rich plasma
}

\author{
- Maczy González¹, Melvis Arteaga-Vizcaíno², Ana Ruiz³, Daniela Esis³, Biagney González³, Ricardo Rosmary Galue³, \\ Nelson Fernández ${ }^{2}$
}

1 Jefe Cátedra de Hematología, Facultad de Medicina, Universidad del Zulia (Venezuela).

2 Instituto de Investigaciones Clínicas, Facultad de Medicina, Universidad del Zulia (Venezuela).

3 Cátedra de Hematología, Facultad de Medicina, Universidad del Zulia (Venezuela).

\section{Resumen}

Objetivo: Evaluar el recuento plaquetario en el plasma rico en plaquetas (PRP) de sujetos sanos antes y después de la administración de antiagregantes plaquetarios.

Material y método: Se estudiaron 32 sujetos, 20 de sexo femenino y 12 de sexo masculino, entre 18 y 50 años, y aparentemente sanos. Se distribuyeron en dos grupos: 1) 16 sujetos que recibieron aspirina (100 mg) y 2) 16 con clopidogrel (75 mg), administrados en dosis única. En cada uno se extrajo 16,5 ml de sangre venosa antecubital para recuento plaquetario en sangre periférica y en PRP, se practicó agregación plaquetaria antes del tratamiento.

Resultados: En todos los sujetos estudiados, se encontró un contaje de plaquetas antes del tratamiento con antiagregantes en sangre periférica de $276,55 \pm 66,43 \times \mathrm{mm}^{3}$ y 24 horas después de 281,38 $\pm 81,55 \times \mathrm{mm}^{3}$. El PRP de los sujetos antes de recibir aspirina fue de 566,86 $\pm 109,16 \times \mathrm{mm}^{3}$ y después de 526,34 $\pm 90,55 \times \mathrm{mm}^{3}$; antes de la ingesta de clopidogrel, fue de $444,76 \pm 100,65 \times \mathrm{mm}^{3}$ y $388,82 \pm 71,94 \times \mathrm{mm}^{3}$ después del tratamiento. Se halló una diferencia significativa para el conteo plaquetario solo para el clopidogrel $(p<0,05)$. La correlación para el conteo de plaquetas antes y después del tratamiento existe solo para aspirina $(r=0,88)$.

Conclusiones: En el presente trabajo, se evidenció una disminución significativa del recuento plaquetario después de administrar clopidogrel, sin correlación antes y después del tratamiento. Son necesarios más estudios que permitan evaluar un mayor número de casos y su efecto sobre la agregación plaquetaria.

Palabras clave: antiagregantes plaquetarios, recuento plaquetario, PRP, aspirina, clopidogrel

\begin{abstract}
Objective: Evaluate the platelet count in the platelets-rich plasma (PRP) from healthy subjects before and after the administration of antiplatelet agents.

Material and method: It is studied 32 subjects, 20 female and 12 male, between 18 and 50 years old and apparently healthy. They were distributed in two groups: 1) 16 subjects who received aspirin (100 mg) and 2) 16 with clopidogrel (75 mg), these doses were unique for a day. In each subject is extracted $16,5 \mathrm{ml}$ of venous blood antecubital for platelet count in peripheral blood and PRP, platelet aggregation before treatment is practiced.

Results: In all the studied subjects are found a platelet count before treatment with antiplatelet in peripheral blood of $276,55 \pm 66,43 \times \mathrm{mm}^{3}$ and 24 hours after $281,38 \pm 81,55 \times \mathrm{mm}^{3}$. In the PRP of subjects before to receiving aspirin was $566,86 \pm 109,16 \times \mathrm{mm}^{3}$ and then $526,34 \pm 90,55 \times \mathrm{mm}^{3}$; prior to clopidogrel ingestion was $444,76 \pm 100,65 \times \mathrm{mm}^{3}$ and $388,82 \pm 71,94 \times \mathrm{mm}^{3}$ after treatment. Significant differences for the platelet count were found only for clopidogrel $(p<0,05)$. The correlation for the count of platelets before and after the treatment only exists for aspirin $(r=0,88)$.

Conclusions: In this study showed a significant decrease in the platelet count after administering clopidogrel without correlation between them before and after treatment. Further studies are necessary to evaluate a larger number of cases and their effect on platelet aggregation.
\end{abstract}

Key words: antiplatelet agents, PRP, platelet count, aspirin, clopidogrel. 


\section{Introducción}

Las plaquetas circulan por la sangre y están involucradas en la hemostasia primaria iniciando la formación de coágulos o trombos, miden aproximadamente de 2 a 4 m y están desprovistas de núcleo, por lo que no se trata de verdaderas células, sino de fragmentos celulares. Su forma fisiológica es discoide, aspecto que se modifica con facilidad por las maniobras de extensión o centrifugación, adquiriendo un aspecto redondeado y emitiendo finas prolongaciones'.

En la superficie plaquetaria, se expresan receptores complejos que son glicoproteínas: el complejo llb-IIla, este receptor se une al fibrinógeno y este sirve para que se unan complejos de glicoproteínas de la superficie de otras plaquetas, contribuyendo con la agregación plaquetaria. Simultáneamente se produce la liberación del contenido de los gránulos de las plaquetas, que liberan: tromboxano, ADP, calcio, entre otros, sustancias que estimulan la agregación plaquetaria. Esta unión es laxa en principio y después se consolida. El proceso finaliza con la activación coordinada de los factores de la coagulación y la consecuente transformación del fibrinógeno en fibrina².

La determinación de las plaquetas en sangre periférica se realiza mediante un examen denominado recuento plaquetario, donde se mide la cantidad de plaquetas por milímetro cúbico, cuyos resultados son determinados por recuento automatizado con equipamiento especializado o por el microscopio. Este recuento tiene como finalidad principal evaluar la eficiencia de la producción de estas células por la médula ósea, así como vigilar el efecto terapéutico. A su vez, ayuda en el diagnóstico de enfermedades que cursan con trombocitopenia y trombocitosis².

Los valores del recuento plaquetario normal se encuentran entre 150.000 a 450.000 plaquetas $/ \mathrm{mm}^{3}$; por debajo de 50.000, se puede producir sangrado espontáneo. Por otra parte, si el número de plaquetas es demasiado alto, pueden formarse coágulos y ocasionar trombosis, los cuales obstruyen los vasos sanguíneos y generan accidente cerebrovascular, infarto agudo de miocardio y embolismo pulmonar, entre otros. Cualquier anormalidad o enfermedad de las plaquetas es denominada trombocitopatía, la cual puede ser provocada por un número reducido de plaquetas (trombocitopenia), por un déficit en la función (tromboastenia) o por un incremento en el número (trombocitosis)33.
La función de las plaquetas es adherirse y agregarse sobre el endotelio, puede ser inhibida por antiagregantes endógenos desencadenados durante la activación plaquetaria y debido a la generación de fibrina, que tiende a limitar la extensión del trombo y a prevenir la coagulación sistémica. Los más importantes inhibidores fisiológicos son la prostaciclina (PG 12), el óxido nítrico, la antitrombina III, la proteína $C$ y el sistema fibrinolítico. De igual manera, se conoce la acción que tienen ciertos fármacos denominados antiagregantes plaquetarios, cuyo efecto principal es inhibir la agregación de las plaquetas y, en consecuencia, la formación de trombos o coágulos en el interior de las arterias y venas. El fármaco antiagregante plaquetario ideal será aquel que inhiba las vías de activación plaquetaria o estimule las de inhibición, o ambas cosas a la vez ${ }^{4}$.

Los fármacos antiagregantes plaquetarios se pueden clasificar según su mecanismo de acción en dos grandes grupos: Ios inhibidores enzimáticos, dentro de los cuales se ubican los inhibidores de la ciclooxigenasa (inhiben la síntesis de tromboxano), como el ácido acetilsalicílico (AAS) o aspirina; y los inhibidores de receptores (inhibidores de receptores de ADP), como la ticlopidina, clopidogrel y prasugrel. La aspirina y el clopidogrel son los fármacos antiagregantes más usados en la prevención de las enfermedades cardiovasculares trombóticas en la medicina actual. La aspirina inhibe la formación de tromboxano A2 en la plaqueta y de prostaciclina en el endotelio vascular. El clopidogrel, droga de la familia de las tienopiridinas, actúa a través de la inhibición de los receptores plaquetarios del ADP4

La aspirina inhibe principalmente a la ciclooxigenasa-1 (COX-1) y con mucho menos afinidad a la ciclooxigenasa-2 (COX-2). La acción de la aspirina produce una acetilación en un residuo de serina del sitio activo de la COX. Con ello impide la metabolización del ácido araquidónico, producto de la acción de las fosfolipasas A2 sobre los fosfolípidos de la membrana plaquetaria, y el resultado final es la disminución casi completa de la formación de tromboxano $A 2$, sustancia proagregante y vasoconstrictora. Como las plaquetas son células anucleadas, la falla en la generación de tromboxano A2 se mantiene durante toda la vida de la plaqueta (8-10 días) $)^{5}$.

EI AAS se administra por vía oral y se absorbe rápidamente por el tracto digestivo. Es hidrolizado 
parcialmente a ácido salicílico durante el primer paso a través del hígado y se distribuye ampliamente por todos los tejidos del organismo. Después de la administración oral y dependiendo de las dosis administradas, se observan salicilatos en plasma a los 5-30 minutos y las concentraciones máximas se obtienen a los 0,25-2 horas. Las concentraciones plasmáticas deben ser por lo menos de $100 \mathrm{mg}$ para obtener un efecto analgésico y se observan efectos tóxicos con concentraciones superiores a $400 \mathrm{mg}$. La aspirina se metaboliza en un $99 \%$ a salicilato y otros metabolitos, y la semivida de eliminación del plasma es de 15 a 20 minutos $^{5}$.

El clopidogrel (C) es una prodroga que requiere la oxidación por el sistema enzimático citocromo P4501 A del hígado para adquirir actividad. Es metabolizado a 2-oxo-clopidogrel, el precursor inmediato del metabolito activo. En condiciones normales solo un 15\% de la dosis administrada se deriva a sus metabolitos con acción sobre los receptores P2Y12 para el ADP, inhibiendo la agregación plaquetaria. El restante 85\% se hidroliza hacia metabolitos inactivos por actividad de las esterasas. Por esta razón, actualmente se incrementa la carga del clopidogrel de 75 a 300-600 mg en situaciones de emergencia, para obtener una mayor cantidad de metabolitos activos y una más rápida acción de estos sobre la función plaquetaria ${ }^{6,7}$. Se utiliza para prevenir episodios arterioscleróticos (infarto de miocardio, ictus y muerte vascular) en pacientes con historia reciente de accidente cerebrovascular, cardio o perivascular. En general, la tolerancia asociada al empleo del clopidogrel es parecida a la de la aspirina, sin embargo, las hemorragias gastrointestinales son menos frecuentes con el primero ${ }^{4}$.

El clopidogrel se administra por vía oral. El compuesto activo es muy lábil y no ha podido ser identificado, por lo que el perfil farmacocinético corresponde al de un metabolito primario inactivo, un ácido carboxílico que supone aproximadamente el $85 \%$ de los metabolitos circulantes en el plasma. La absorción del clopidogrel es del $50 \%$ y no es afectada significativamente por los alimentos. Las concentraciones plasmáticas máximas del metabolito primario se consiguen una hora después de la dosis de $75 \mathrm{mg}$. Dos horas después de una dosis oral, ya no son detectables concentraciones plasmáticas del fármaco sin alterar. El clopidogrel y su principal metabolito se unen de forma reversible a las proteínas plasmáticas (98 y 94\%, respectivamente). Con dosis repetidas de $75 \mathrm{mg}$ por día, el máximo de inhibición de la agregación plaquetaria se consigue a los 3-7 días. En condiciones de equilibrio, la agregación plaquetaria es inhibida en un $40-60 \%{ }^{4}$.

Pocos trabajos de investigación se han llevado a cabo sobre el efecto de los antiagregantes plaquetarios en el recuento plaquetario, entre ellos se encuentra el de Hayasaka y colaboradores en el 2013, quienes realizaron un estudio comparativo de la alteración de los parámetros hematológicos en dos grupos de pacientes tratados con aspirina + clopidogrel y aspirina solamente, durante dos meses. Estos investigadores no hallaron diferencias significativas en el conteo plaquetario (basal y postratamiento), pero sí para otros parámetros hematológicos ${ }^{6}$.

En tanto, Cohen y colaboradores, en el 2012, observaron una disminución inicial del recuento plaquetario en pacientes sometidos a cirugía de revascularización miocárdica, luego de 72 horas de la cirugía y del inicio del tratamiento con AAS, con un posterior ascenso plaquetario siete días después?

Es por ello por lo que el estudio de la acción de los antiagregantes plaquetarios y su relación con el recuento plaquetario es de gran relevancia, ya que permitirá inferir si estos antiagregantes alteran o no el recuento plaquetario en el PRP del paciente, $y$, de esta manera, se pueda valorar adecuadamente el efecto terapéutico del fármaco empleado. De allí que los objetivos planteados en este trabajo de investigación fueron los siguientes:

\section{Objetivo general}

Evaluar el recuento plaquetario en el PRP de sujetos sanos antes y después de la administración de antiagregantes plaquetarios.

\section{Objetivos específicos}

- Determinar el recuento plaquetario en el PRP de sujetos sanos que asisten al Instituto de Investigaciones Clínicas Dr. Américo Negrette de la Facultad de Medicina, de la Universidad del Zulia (LUZ), antes y 24 horas después de la ingestión de antiagregantes plaquetarios (aspirina y clopidogrel).

- Relacionar el recuento plaquetario en el PRP antes y después de la administración de antiagregantes plaquetarios (AAS o clopidogrel). 


\section{Material y método}

\section{Tipo y diseño de investigación}

De acuerdo con los propósitos inmediatos que persiguen los autores de la investigación, esta se ubica en un nivel de estudio de corte transversal analítico ${ }^{8}$.

\section{Población y muestra}

La población objeto de estudio estuvo conformada por 80 sujetos adultos, de ambos sexos, aparentemente sanos, que acudieron de manera voluntaria al Servicio de Hematología del Instituto de Investigaciones Clínicas (IIC) Dr. Américo Negrette de la Facultad de Medicina de la LUZ, durante el período del $1^{\circ}$ de septiembre al 15 de diciembre del 2012.

La muestra que se consideró en esta investigación fue por conveniencia, en la cual se realizó la selección de los elementos con base en criterios o juicios del investigador ${ }^{9}$. En ese sentido, la muestra que se seleccionó de la población antes descrita representó el $40 \%$, 32 sujetos (20 de sexo femenino y 12 de sexo masculino), que correspondió solo a aquellos sujetos que cumplieron con los criterios de selección que se describen a continuación.

\section{Criterios de selección}

Se tomaron en cuenta los siguientes:

\section{Inclusión:}

- Edad entre 18 y 50 años.

- En ayunas.

- Sin enfermedad clínica de base conocida.

- Aparentemente sanos.

- Estudios de hemostasia y agregación plaquetaria normales.

\section{Exclusión:}

- Haber ingerido antiagregantes plaquetarios 11 días antes del estudio.

- Haber ingerido bebidas alcohólicas 10 días antes del estudio.

- Estar ingiriendo cualquier tipo de tratamiento.

A todos ellos se les requirió consentimiento informado por escrito, el cual contó con la aprobación del comité de ética de la Institución y se procedió de acuerdo con los principios de la Declaración de Helsinki de 1975 (actualizada en el 2000) y con las recomendaciones elaboradas por el Consejo de Organizaciones Internacionales de Ciencias Médicas (CIOMS) en el 2002.

\section{Procedimiento de laboratorio}

Para el procesamiento de las muestras, se empleó un contador automático de células sanguíneas (Beckman Coulter AC-T USA), recogiendo los datos concernientes a plaquetas y glóbulos blancos. La agregación se efectuó según el método turbidimétrico de Born ${ }^{10}$, empleando un agregómetro (Chrono-log Corp. Haverton, PA, USA). El resultado obtenido se expresó en porcentaje.

Todos los sujetos fueron sometidos a un examen clínico y de laboratorio exhaustivo, con el fin de descartar enfermedades sistémicas. Estos se distribuyeron de la siguiente manera:

- Treinta y dos sujetos sin tratamiento previo con drogas antiagregantes plaquetarias.

- Se dividieron a su vez en dos subgrupos:

- Uno constituido por 16 sujetos que recibieron aspirina a una dosis de $100 \mathrm{mg}$, una sola por un día.

- El segundo por el resto de los 16 individuos, a quienes se les dio clopidogrel a una dosis de 75 mg en dosis única un solo día.

A 32 sujetos sin tratamiento, en ayunas, previa asepsia de la zona, se les extrajo $16,5 \mathrm{ml}$ de sangre venosa antecubital, utilizando mariposas $N^{\circ} 21$ y empleando la técnica de la doble jeringa para evitar la activación de las plaquetas:

1. La primera jeringa contenía $2,5 \mathrm{ml}$ de sangre venosa que se dispensó en tubos de vidrio que tenían EDTA para estudio de hematología, y se recogieron los datos concernientes a plaquetas y glóbulos blancos, empleando un contador automático de las células sanguíneas (Beckman Coulter AC-T USA).

2. La segunda jeringa contenía $5 \mathrm{ml}$ de sangre venosa, que se procesó para la aplicación del protocolo de agregación plaquetaria, como se describe en texto suplementario anexo.

a) $9 \mathrm{ml}$ de la muestra restante se colocaron en otro tubo plástico que contenía $1 \mathrm{ml}$ de citrato de sodio al 3,8\% (9/1), dejando reposar 20 minutos, y luego fueron sometidos a centrifugación, en centrífuga clínica a una velocidad de 1.400 r. p. m. por 7 minutos a $267 \mathrm{G}$ y siguiendo la técnica de Anitua ${ }^{11}$ para la obtención de PRP. Del volumen total obtenido en el PRP, se extrajeron 0,5 ml, que corresponden a plasma pobre en plaquetas (PPP) que se descartó. Al PRP obtenido se le hizo el recuento plaquetario en el contador hematológico automatizado. 
b) Al sujeto se le instruyó para que antes de consumir el desayuno ingiriese la dosis oral correspondiente al antiagregante respectivo (AAS $100 \mathrm{mg}$ o clopidogrel $75 \mathrm{mg}$ ); y a las 24 horas se le extrajo $9 \mathrm{ml}$ de sangre venosa antecubital, empleando mariposas $N^{\circ} 21 \mathrm{y}$ siguiendo la técnica de la doble jeringa para evitar la activación de las plaquetas. Estos se colocaron en otro tubo plástico que contenía $1 \mathrm{ml}$ de citrato de sodio al 3,8\% (9/1), dejando reposar 20 minutos, y luego fueron sometidos a centrifugación, en centrífuga clínica a una velocidad de 1.400 r. p. m. por 7 minutos a $267 \mathrm{G}$ y siguiendo la técnica de Anitua ${ }^{11}$ para la obtención de PRP, al cual se le realizó el conteo plaquetario respectivo.

c) Del volumen total obtenido en el PRP, se extrajeron previamente $0,5 \mathrm{ml}$, que corresponden a plasma pobre en plaquetas (PPP) que se descartó.

\section{Valores referenciales}

Se consideran los valores referenciales para plaquetas entre 150.000 a $450.000 \times$ mm $^{3}$ en sangre periférica $^{12}$, mientras que, para cada agonista plaquetario utilizado, se consideró lo descrito en la literatura, a saber: el 68-88\% para ADP, el 70-94\% para el colágeno y para epinefrina el $78-88 \%{ }^{13}$.

\section{Técnicas de procesamiento y análisis de datos}

Para la tabulación y el análisis de los resultados obtenidos, se utilizó la estadística respectiva. Los datos se muestran en tablas y gráficos, en valores absolutos y porcentajes, así como media +/- 1 desviación estándar. Para establecer la correlación entre el número de plaquetas obtenido y el tipo de fármaco antiagregante administrado en los grupos de estudio respectivos (antes y después de la administración del fármaco), se empleó la correlación de Pearson, usando $p<0,05$ como la menor probabilidad estadística ${ }^{14}$.

\section{Resultados}

En el presente estudio, de los 32 sujetos contemplados, 1 presentó cifras de plaquetas de 120 × 109/I, por lo que fue excluido y remitido a especialista para su estudio y análisis. El total de la muestra quedó finalmente en 31 sujetos.

En la tabla 1, se expresan los valores promedios y desviación estándar del recuento plaquetario en sangre periférica y en el PRP obtenido en los 31 sujetos sanos
Tabla 1. Valores promedios y desviación estándar del recuento plaquetario en las diferentes muestras de los sujetos sanos estudiados antes y después del tratamiento con antiagregante plaquetario en el Instituto de Investigaciones Clínicas Dr. Américo Negrette, LUZ, 2012

\begin{tabular}{|c|c|c|c|}
\hline \multirow{2}{*}{$\begin{array}{c}\text { Plaquetas } \\
\left(x \mathrm{~mm}^{3}\right)\end{array}$} & \multicolumn{2}{|c|}{ Promedio \pm desviación estándar } & \multirow[b]{2}{*}{$\mathbf{p}$} \\
\hline & Antes & Después & \\
\hline $\begin{array}{l}\text { Plaquetas sangre periférica } \\
\qquad n=31\end{array}$ & $276,55 \pm 66,43$ & $281,38 \pm 81,55$ & NS \\
\hline $\begin{array}{l}\text { PRP AAS } \\
\mathrm{n}=15\end{array}$ & $566,86 \pm 109,16$ & $526,34 \pm 90,55$ & NS \\
\hline $\begin{array}{l}\text { PRP clopidogrel } \\
n=16\end{array}$ & $444,76 \pm 100,65$ & $388,82 \pm 71,94$ & $p<0,05$ \\
\hline
\end{tabular}

objeto de estudio antes y después del tratamiento con antiagregante plaquetario y analizados en la Sección de Hematología del IIC, Facultad de Medicina, LUZ. Se observa que, al comparar los valores del conteo plaquetario en sangre periférica antes y después del tratamiento con AAS y/o clopidogrel, no se encontraron diferencias estadísticamente significativas (NS), lo mismo se observó con las cifras plaquetarias en el PRP antes y después de la administración de AAS (NS). Se notó que existe diferencia estadísticamente significativa solo para el clopidogrel $(p<0,05)$.

En la tabla 2, se muestra la correlación existente entre el recuento plaquetario antes y 24 horas después de la ingestión de AAS o clopidogrel. La correlación existe solo para el AAS $(r=0,88)$, para el clopidogrel fue de 0,37 .

Tabla 2. Correlación del recuento plaquetario de los sujetos sanos estudiados tratados con AAS y clopidogrel, antes y 24 horas después de su ingestión

\begin{tabular}{|c|c|c|c|}
\hline \multirow{2}{*}{$\begin{array}{c}\text { Plaquetas } \\
\left(x \mathrm{~mm}^{3}\right)\end{array}$} & \multicolumn{2}{|c|}{ Promedio \pm desviación estándar } & \multirow{2}{*}{$\begin{array}{l}\text { Correlación } \\
\text { (r) }\end{array}$} \\
\hline & Antes & Después & \\
\hline $\begin{array}{c}\text { Con AAS } \\
n=14\end{array}$ & $566,86 \pm 109,16$ & $526,35 \pm 90,55$ & 0,88 \\
\hline $\begin{array}{c}\text { Con clopidogrel } \\
n=17\end{array}$ & $444,76 \pm 100,65$ & $388,82 \pm 71,94$ & 0,37 \\
\hline
\end{tabular}

PRP: plasma rico en plaquetas. AAS: aspirina. n: número de individuos. r: correlación. Fuente: Sección de Hematología, IIC, Facultad de Medicina, LUZ.

\section{Discusión}

En la población objeto de estudio, el promedio de plaquetas en sangre periférica fue de 276,55 \pm $66,43 \times 10^{9} /$ antes de la administración del fármaco antiagregante, y 24 horas después de su ingestión (tabla 1), se encontró un ligero incremento que no fue estadísticamente significativo $\left(281,38 \pm 81,55 \times 10^{9} / l\right)$. Caso contrario se observó en las muestras contenidas en los tubos con citrato de sodio para obtener PRP, donde se halló un valor de 566,86 \pm 109,16 x 10\%/l antes de ingerir AAS y $444,76 \pm 100,65 \times 10^{9} / I$ antes de la administración de clopidogrel. Solo para este último, la cifra de plaquetas disminuyó de manera significativa 
$(p<0,05)$ después de su ingestión $(444,76 \pm 100,65$ x109 vs. $\left.388,82 \pm 71,94 \times 10^{9} / 1\right)$.

Es importante acotar que los sujetos de este trabajo son personas sanas, en quienes el recuento plaquetario se realizó antes y al día siguiente de la administración del fármaco antiagregante; estas personas no estuvieron sometidas a ningún procedimiento invasivo y no presentaban procesos de inflamación. En estas condiciones, no existe la presencia de factores que pudieran afectar el número de plaquetas, tal como ocurre en procesos inflamatorios o infecciosos que condicionan trombocitosis secundarias ${ }^{15,16}$

Al respecto, se reporta que el AAS ocasiona una producción de plaquetas de 5,5 x 107 células/día17, lo que pudiera explicar el discreto aumento que se observó en el recuento plaquetario el día siguiente solo en sangre periférica. No obstante, otros autores describen un incremento mayor al nuestro, justificado por un aumento acelerado de la producción de plaquetas conocido como recambio (turnover) plaquetario, esto ocurre en pacientes sometidos a cirugía cardíaca en la primera semana del período posoperatorio, atribuyéndosele al estímulo trombopoyético que provocan las citocinas inflamatorias que se liberan después de estos procedimientos ${ }^{17,18,19}$.

Con respecto al AAS, se describe una relación entre el recuento plaquetario y los valores de agregación plaquetaria en el posoperatorio de cirugía de revascularización miocárdica tratada con este antiagregante. Este efecto es igual a los que presentan grupos de sujetos sin este medicamento, pero con la misma condición patológica ${ }^{7}$, sugiriendo que, aunque varíe el número de plaquetas, el efecto de los diferentes agonistas sobre su agregación no se modifica.

En la presente investigación, la cifra de plaquetas disminuyó de manera no significativa 24 horas después de la administración del AAS, pero se mantuvo en el rango normal para la realización de agregación plaquetaria. Estos hallazgos discrepan de los obtenidos por Hayasaka y colaboradores en el 2013, quienes llevaron a cabo un estudio comparativo de la afectación de los parámetros hematológicos en dos grupos de pacientes tratados por dos meses con aspirina + clopidogrel y aspirina solamente, durante dos meses. Estos investigadores no encontraron diferencias significativas en el conteo plaquetario (basal y postratamiento), pero sí para la cuenta roja, blanca, hemoglobina y hematocrito ${ }^{6}$.
Los resultados del presente estudio permiten inferir que el AAS pareció afectar el recuento plaquetario discretamente, lo cual merece estudios posteriores con un mayor número de muestras y en un período más prolongado de tratamiento.

Así mismo, se observó una disminución de la cifra de plaquetas en los sujetos tratados con clopidogrel, siendo su función principal bloquear el receptor de ADP conocido como P2Y e interferir con el receptor plaquetario del fibrinógeno (gllb-IIla), impidiendo la vía de mayor amplificación de la agregación plaquetaria, lo cual pudiese haber condicionado la alteración de las cifras plaquetarias 20,21 .

En ese orden de ideas, se encontró una correlación $(r=0,88)$ entre el recuento plaquetario antes y después de la administración de aspirina. Esto significa que, aunque varíe, este conteo no induce alteración sobre la respuesta esperada; no así para el clopidogrel (tabla 2), donde no se observó correlación entre estos dos elementos $(r=0,37)$. Esto sugiere que pudiese afectarse la respuesta de las plaquetas ante los agonistas plaquetarios, lo que habría de investigarse. No obstante, es importante destacar que el recambio plaquetario en condiciones normales después de la administración de antiagregante es de aproximadamente siete días ${ }^{15}$.

En resumen, el presente trabajo permitió evaluar el recuento plaquetario en el PRP de sujetos sanos antes y después de la administración de antiagregantes plaquetarios, encontrándose una disminución significativa solo para el clopidogrel 24 horas después de su administración. Los datos aquí mostrados son de interés clínico, pues permiten conocer cómo estos medicamentos de uso frecuente influyen en el cambio del recuento plaquetario, permitiendo al facultativo tomar las previsiones necesarias para evitar un efecto indeseado, como trombosis o hemorragia, que representan los dos efectos extremos no deseados. Son necesarios más estudios que permitan evaluar un mayor número de casos y su efecto sobre la agregación plaquetaria.

\section{Agradecimiento}

Esta investigación fue financiada por el Consejo de Desarrollo Científico y Humanístico de la Universidad del Zulia (Condes), a través del proyecto VAC-CondesCC-0019-12. Los autores agradecen la dotación de materiales reactivos y equipo necesario para la ejecución 
de este trabajo de investigación. Así mismo, se contó con la colaboración del Laboratorio de Hematología del Instituto de Investigaciones Clínicas Dr. Américo Negrette de la Facultad de Medicina de la Universidad del Zulia, en cuyos laboratorios se llevó a cabo el análisis de las muestras recolectadas.

\section{Referencias}

1. Campbell NA. Biología. 8a ed. Londres: Editorial Pearson; 2008.

2. Hilman R, Kenneth $A$, Henry M. Hematología en la práctica clínica. $4^{\mathrm{a}}$ ed. Editorial Manual Moderno; 2005.

3. Patrono C, Bachmann F, Baigent C, Bode C, De Caterina R, Charbonnier B et ál. Grupo de trabajo sobre el uso de agentes antiplaquetarios en pacientes con enfermedad cardiovascular aterosclerótica de la Sociedad Europea de Cardiología. Documento de consenso de expertos sobre el uso de agentes antiplaquetarios. Rev Esp Cardiol. 2004;57:963-80.

4. Harker LA, Boissel JP, Pilgrim AJ, Gent M. Comparative safety and tolerability of clopidogrel and aspirin: results from CAPRIE. CAPRIE Steering Committee and Investigators. Clopidrogel versus aspirin in patients at risk of ischaemic events. Drug Saf. 1999;21(4):325-35

5. Tanasescu S, Lévesque H, Thuillez C. Farmacología de la aspirina. Rev Med Int. 2000;21:(1):18s-26s.

6. Hayasaka M, Takahashi Y, Nishida Y, Yoshida Y, Hidaka S, Asai S. Comparative effect of clopidogrel plus aspirin and aspirin monotherapy on hematological parameters using propensity score matching. Vasc Health Risk Manag. 2013;9:65-70.

7. Cohen Arazimtsac $H$, Carnevalini M, Falconi $E$, Ovejero R, Giorgimtsac M, Carolimtsac C et ál. Relación entre el efecto antiagregante de la aspirina y el recuento plaquetario. Posibles implicaciones en la dosificación. Rev Argent Cardiol. 2012;80:114-20.

8. Hernández R, Fernández C, Baptista LP. Metodología de la investigación. $4^{a}$ ed. Iztapalapa-México D.F.: Editorial McGrawHill; 2006.
9. Arias FG. El proyecto de investigación. Introducción a la metodología científica. 5a ed. Caracas: Editorial Episteme; 2006.

10. Born GV. Aggregation of blood platelets by adenosine and its reversal. Nature. 1962;194:927-9.

11. Anitua E. La utilización de los factores de crecimiento plasmáticos en cirugía oral, maxilofacial y periodoncia. RCOE. 2001;6(3):305-15.

12. Ware JA, Coller BS. Platelet morphology, biochemistry and function. In: Beutler E, Lichtman MA, Coller BS, editors. Williams hematology. 5th ed. New York: McGraw-Hill; 1995. p. 1161-201

13. Bick RL. Platelet function defects. In: Bick RL, editor. Disorders of thrombosis and hemostasis: clinical and laboratory practice. USA: Lippincott Williams \& Wilkins; 2002. p. 59-90.

14. Daniel W. Estadística con aplicaciones a las ciencias sociales y la educación. México, D.F.: McGraw-Hill; 1988.

15. Molina V, Arruzazabala L, Carbajal D, Más R. Farmacología de los agentes antiagregantes plaquetarios. Revista Cenic Ciencias Biológicas. 2005;36(1):3-12.

16. Buss DH, Cashell AW, $\mathrm{O}^{\prime}$ Connor ML, Richards $\mathrm{F}$ 2nd, Case LD. Occurrence, etiology, and clinical significance of extreme thrombocytosis: a study of 280 cases. Am J Med. 1994;96(3):247-53

17. Bizzozero J. On a new blood particle and its role in thrombosis and blood coagulation. Virchows Arch Pathol Anat Physiol Clin Med. 1882;90:261-332.

18. Folman $C C$, Ooms $M$, Kuennen BB, De Jong SM, Vet RJ, de Haas $M$, et al. The role of thrombopoietin in post-operative thrombocytosis. Br J Haematol. 2001;114(1):126-33.

19. Kaser A, Brandacher G, Steurer W, Kaser S, Offner FA, Zoller $\mathrm{H}$, et al. Interleukin- 6 stimulates thrombopoiesis through thrombopoietin: role in inflammatory thrombocytosis. Blood. 2001;98(9):2720-5

20. Blair P, Flaumenhaft R. Platelet alpha-granules: basic biology and clinical correlates. Blood Rev. 2009;23(4):177-89.

21. Schrör K. The basic pharmacology of ticlopidine and clopidogrel. Platelets. 1993;4(5):252-61. 


\section{Anexos}

\section{Técnica de agregometría}

La segunda jeringa contenía $5 \mathrm{ml}$ de sangre venosa, que se procesó como se describe a continuación:

$4,5 \mathrm{ml}$ se dispensaron en un tubo plástico que contenía $0,5 \mathrm{ml}$ de citrato de sodio al 3,8\% y se centrifugó a 800 r. p. m. (180 g) durante 10 minutos a temperatura ambiente para obtener PRP; luego, el remanente de cada muestra se centrifugó a 4.500 r. p. m. durante 20 minutos, en centrífuga refrigerada (Sorvall), para obtener PPP. Para realizar la agregación plaquetaria, la concentración de plaquetas se ajustó a 250 x 109/I con el PPP. La prueba de agregación en las muestras se efectuó utilizando $0,45 \mathrm{ml}$ de PRP y se le adicionaron los siguientes inductores: ADP (adenosina 5-difosfato): 5 x 10-6 M (concentración final: 2,14 $\mu \mathrm{g} / \mathrm{ml})$, colágeno: 1,2 x 10-2 M (concentración final: $3 \mu \mathrm{g} / \mathrm{ml})$, epinefrina: 0,6 x 10-4 M (concentración final: $11 \mu \mathrm{g} / \mathrm{ml}$ ). La agregación se llevó a cabo según el método turbidimétrico de Born10, empleando un agregómetro
Chrono-log (Corp. Haverton, PA, USA). El resultado conseguido se expresó en porcentaje.

\section{Agregación plaquetaria}

Una vez obtenida la muestra de sangre venosa y cuantificadas las plaquetas en la primera centrifugación, se realizó el ajuste a $250.0000 \times \mathrm{mm}^{3}$ por $\mu \mathrm{l}$, de la siguiente manera:

1. Se determinó un factor de dilución:

$$
\mathrm{F}=\mathrm{N}^{\circ} \text { de plaquetas en el PRP/250.000 }
$$

2. Se dividió el volumen deseado con el que se va a trabajar entre el factor de dilución:

$$
2,5 \mathrm{ml} \text { de PRP/F }
$$

3. La cantidad obtenida en el paso anterior se completó con el PPP conseguido en la segunda centrifugación, hasta completar los 2,5 ml deseados.

4. Una vez efectuado el ajuste, se procedió a hacer la agregación plaquetaria, utilizando $1 \mathrm{ml}$ de la preparación (ajuste) para cada agregante plaquetario y 0,45 $\mathrm{ml}$ del agregante.

5. Se llevó a cabo la lectura en el agregómetro. 